\title{
QUALIDADE FÍSICA E QUÍMICA DO SOLO EM ÁREAS DE EXPLORAÇÃO FLORESTAL NO MATO GROSSSO ${ }^{1}$
}

\author{
Alice Aparecida Iarema², Luiz Eduardo Ferreira Fonte ${ }^{3}$, Raphael Bragança Alves Fernandes ${ }^{3}$, Carlos \\ Ernesto Gonçalves Reynaud Schaefer ${ }^{3}$ e Luiz Carlos Pereira ${ }^{4}$
}

\begin{abstract}
RESUMO - Com o objetivo de avaliar o impacto provocado nos solos sob pátios de estocagem de madeira, bem como a influência do formato dos pátios sobre o processo de degradação nesses locais, foram selecionados cinco pátios em uma área de exploração de madeira, onde predominava solo do tipo Latossolo VermelhoAmarelo, localizada no Município de Nova Maringá, MT. Foram escolhidos quatro pátios de formato quadrado e um de formato retangular. Em cada pátio, estabeleceram-se três transectos, que adentraram a mata nativa, sendo feitas coletas de solo na profundidade de 0 a $20 \mathrm{~cm}$, em cinco pontos de cada transecto. Essas amostras foram utilizadas para a determinação de textura, argila dispersa em água, umidade na capacidade de campo, estabilidade e distribuição de agregados, análises químicas de rotina, C-total, N-total e C mineralizável. Para a determinação da densidade do solo e densidade de partículas, as amostras foram retiradas nas profundidades de 0 a $5 \mathrm{~cm}$ e 15 a $20 \mathrm{~cm}$ do transecto central. Os resultados foram submetidos a testes de média (Tukey, $\mathrm{P}<0,05)$. Testes de resistência à penetração em cinco pontos de cada pátio e três pontos na mata adjacente foram realizados na época da seca e na época chuvosa. Os resultados mostraram que houve forte degradação nas propriedades dos solos sob pátios de estocagem, observando-se expressivo aumento na densidade do solo e resistência à penetração, bem como diminuição da porosidade total, do DMP dos agregados e dos teores de nutrientes. A utilização de pátios com diferentes formas não minimizou os impactos ambientais.
\end{abstract}

Palavras-chave: Pátios de estocagem, Impactos ambientais e Física do solo.

\section{PHYSICAL AND CHEMICHAL QUALITY IN SOILS IN FOREST EXPLOITATION AREAS IN MATO GROSSO}

\begin{abstract}
The objective of this work was to evaluate the impact in soils under wood storage clearings as well as the influence of clearing shape on the degradation process in these sites. Thus, it was selected five wood storage clearing areas in a logging exploitation area, where the predominant type of soil is RedYellow Latosol, in Nova Maringa-MT. Four squared clearing storage areas a rectangular one were selected. In each clearing, three transects that were within the native forest were established, and a sample collection of soil at 0-20 cm layer was made in five points in each transect. These samples were used for determination of texture, water-dispersible clay, moisture in field capacity, aggregate distribution and stability, chemical analyses, total carbon, total nitrogen and mineralizable carbon. For soil and particle density measurements, samples were taken from 0-5 cm and 15-20 cm layers in the central transect. Results were submitted to statistical tests (Tukey test, $P<0.05$ ). Penetration resistance tests at five different points in each clearings, and three points in the neighbor forest were carried out in the dry and wet season. The results indicate a strong degradation of soils under wood-storage clearings with a significant increase in soil density and penetration resistance as well as a reduction in total porosity, in the aggregate diameters and in the nutrient levels. The use of wood-storage clearings with different shapes did not decrease the environment impacts.
\end{abstract}

Keywords: Environment impacts, Soil physics and Wood-storage clearings

\footnotetext{
${ }^{1}$ Recebido em 14.12.2007 e aceito para publicação em 18.04.2011.

${ }^{2}$ Professora do Ensino Fundamental da rede estadual de Mato Grosso. E-mail: <alirema3@yahoo.com.br>.

${ }^{3}$ Departamento de Solos, Universidade Federal de Viçosa, UFV, Brasil. E-mail: <luizfontes@ufv.br>, <raphael@ufv.br> <carlos.schaefer@ufv.br>.

${ }^{4}$ Universidade Federal do Mato Grosso, Faculdade de Agronomia e Medicina Veterinária, Departamento de Solos e Engenharia Rural, Cuiabá, MT, Brasil. E-mail: <lcpgeop@gmail.com>.
} 


\section{INTRODUÇÃO}

O Brasil é um país que se destaca no cenário da exploração florestal mundial. A participação do setor florestal no mercado internacional se mostra relevante para a economia brasileira, por meio dos fluxos de capital, trabalho e produtos associados aos efeitos alocativos da atividade florestal (VALVERDE et al., 2003). No Estado de Mato Grosso, a atividade madeireira contribui em grande parte para o desenvolvimento econômico de vários municípios, principalmente na região médionorte do Estado. No entanto, a falta de planejamento e o manejo inadequado já provocaram o esgotamento da madeira em diversas regiões. Mesmo em áreas manejadas, impactos têm sido causados por essa atividade não só sobre os recursos florestais, mas também sobre o solo.

Uma das etapas do processo exploratório da madeira consiste na construção de pátios de estocagem, conhecidos também como esplanadas, que consistem de pequenas clareiras abertas dentro da floresta para concentrar as toras arrastadas pelos tratores, alcançando situações críticas de compactação nessas áreas.

A compactação resulta em aumento da densidade do solo, modificando outras características físicas que, por sua vez, afetam o desenvolvimento vegetal, em virtude das alterações que provocam no ambiente radicular. A redução do espaço poroso, com consequente diminuição nas taxas de difusão de oxigênio, a redução da quantidade de água disponível e o incremento da resistência do solo à penetração são exemplos dessas modificações que podem limitar o crescimento das plantas. O sistema de manejo do solo altera as propriedades físicas e mecânicas do solo com diferentes níveis de compactação, em razão do teor de água, das diferentes classes de solos e da época de realização das operações mecanizadas (KONDO; DIAS JÚNIOR, 1999).

Em áreas de exploração de madeira, os pátios de estocagem são os locais que sofrem os maiores impactos ambientais, principalmente no que se refere às propriedades físicas do solo, já que são áreas que recebem uma grande carga, levando a aumento expressivo da compacidade do solo nessas áreas. As alterações causadas na estrutura do solo dificultam a regeneração da vegetação natural nessas clareiras, que estando descobertas ficam sujeitas a processos erosivos.
Os objetivos deste trabalho foram: 1) avaliar o impacto provocado nos solos sob esplanadas e o seu processo de recuperação natural, por meio de alguns indicadores físicos e químicos de qualidade do solo; e 2) analisar a influência do formato das esplanadas sobre o processo de degradação dessas áreas.

\section{MATERIAL E MÉTODOS}

O estudo foi realizado na Fazenda APASA, localizada entre as coordenadas geográficas 560’ $10^{\prime \prime}$ e $57^{\circ} 17^{\prime} 55^{\prime \prime}$ de longitude oeste e $12^{\circ} 18^{\prime} 31^{\prime \prime}$ e $12^{\circ} 35^{\prime} 13^{\prime \prime}$ de latitude sul, no Município de Nova Maringá, microrregião de Arinos, médio-norte do Estado de Mato Grosso. A fazenda é uma área de exploração de madeira nativa de 50.000 ha, certificada pelos padrões do Forest Stewardship Council, Inglaterra (FSC), onde predomina o Latossolo Vermelho-Amarelo distrófico, com histórico de exploração florestal iniciado no ano de 1995.

Conforme classificação de Köppen (1948), o clima da região é caracterizado como tropical (Aw), possuindo duas estações bem definidas, sendo com período chuvoso de outubro a abril e precipitação média anual entre $1.800 \mathrm{~mm}$ e $2.200 \mathrm{~mm}$.

Para a coleta das amostras de solo foram escolhidos cinco pátios de estocagem (esplanadas), sendo quatro pátios de formato quadrado (20 m x 20 m): A (área 1) utilizada em 1995 no período chuvoso; A (área 2) utilizada em 1997 no período de estiagem; A (área 4) utilizada em 2000 no período chuvoso; e $A_{5}$ (área 5) utilizada em 2003 no período de estiagem e um pátio retangular (12 m x 30 m): A (área 3) utilizada em 2003 no período de estiagem. Amostras de solo foram também coletadas em áreas de mata próxima a cada pátio de estocagem selecionado, para fins de referência e discussão dos resultados. Em cada área (A) foram nomeadas $\mathrm{E}$ as esplanadas e $\mathrm{M}$ as matas adjacentes às esplanadas.

Cada esplanada foi utilizada apenas uma vez, na época citada, por um período aproximado de 30 dias, durante a retirada da madeira do talhão, sendo depois abandonada sem nenhuma intervenção. No momento da coleta, apenas a presença de algumas plantas pioneiras foi observada.

Todas as amostras deformadas de solo foram coletadas no mês de fevereiro de 2004 e as amostras indeformadas, no mês de agosto do mesmo ano. Para a coleta foram estabelecidos três transectos em cada esplanada selecionada, distribuídos de forma 
equidistante ao longo de sua extensão, e considerou-se ainda a floresta nativa adjacente em ambos os lados. Em cada transecto foram estabelecidos cinco pontos para a coleta das amostras (três pontos dentro da esplanada e dois pontos na floresta nativa), sendo abertas três pequenas trincheiras em cada ponto. Nos pontos de coleta nas áreas de floresta foi estabelecida uma distância de $20 \mathrm{~m}$ a partir da borda da esplanada.

Com a utilização de anel de aço (Kopecky) de bordas cortantes, amostras indeformadas foram retiradas, em triplicatas, em todos os pontos do transecto central de cada área, nas profundidades de 0 a $5 \mathrm{~cm}$ e 15 a $20 \mathrm{~cm}$. Amostras deformadas foram coletadas em todos os pontos de todos os transectos, na profundidade de 0 a $20 \mathrm{~cm}$, formando-se uma amostra composta para cada ponto a partir das três trincheiras. Parte da amostra composta, de tamanho entre 4 e $2 \mathrm{~mm}$, foi utilizada para determinação da estabilidade de agregados e a outra parte, que passou por peneira de $2 \mathrm{~mm}$, constituiu a terra fina seca ao ar (TFSA), que foi utilizada para a realização de análises físicas e químicas. Em todos os pontos de todos os transectos, na profundidade de 0 a $20 \mathrm{~cm}$, coletaram-se amostras de solo que foram mantidas a uma temperatura de $\pm 4{ }^{\circ} \mathrm{C}$, utilizadas para determinação do carbono mineralizável.

As análises físicas efetuadas foram: textura, argila dispersa em água, densidade do solo (método do anel volumétrico), densidade de partículas (método do balão volumétrico), porosidade total, umidade na capacidade de campo em função do equivalente de umidade, diâmetro médio ponderado (DMP) dos agregados e umidade do solo. Todas as análises foram executadas de acordo com Embrapa (1997), à exceção da textura e capacidade de campo, efetuadas conforme adaptações de Embrapa (1997), segundo Ruiz (2003) e Ruiz et al. (2003), respectivamente.

Os testes de resistência à penetração (RP) foram realizados em duas épocas: fevereiro de 2004 (estação chuvosa) e agosto de 2004 (estação seca), utilizando-se um penetrômetro de impacto, modelo IAA/ PLANALSUCAR STOLF. Foram avaliados oito pontos por área estudada, dos quais três pontos se encontravam na mata adjacente e cinco dentro da esplanada. Ao redor de cada ponto foram efetuadas três avaliações de RP.
As análises químicas de rotina foram conduzidas de acordo com Defelipo e Ribeiro (1981). A determinação do carbono orgânico total (COT) foi realizada segundo Yeomans e Bremner (1988) e a determinação do nitrogênio total (NT), feita pelo método de Kjeldahl (EMBRAPA, 1997). O carbono mineralizável, quantificado a partir da evolução de $\mathrm{CO}_{2}$, foi efetuado conforme a metodologia proposta por Gregorich et al. (1990), com adaptações de Lima (1996).

Todas as análises físicas e químicas foram realizadas em laboratórios do Departamento de Solos da Universidade Federal de Viçosa (UFV), em Viçosa, MG.

A análise estatística dos dados foi efetuada utilizando-se o programa SAEG (Sistema para Análises Estatísticas), em que os resultados foram submetidos a testes de média (Tukey, $\mathrm{P}<0,05$ ).

\section{RESULTADOS E DISCUSSÃO}

As áreas em estudo apresentam solos de textura média, com teores de argila variando de $192 \mathrm{~g} \mathrm{~kg}^{-1} \mathrm{a}$ $320 \mathrm{~g} \mathrm{~kg}^{-1}$ (Tabela 1). Os valores de umidade na capacidade de campo (UCC) ficaram entre 0,16 e $0,22 \mathrm{~kg} \mathrm{~kg}^{-1}$. As áreas $\mathrm{E}_{5}$ e $\mathrm{M}_{5}$ apresentaram os maiores valores $(0,22$ e $0,21 \mathrm{~kg} \mathrm{~kg}^{-1}$, respectivamente), resultado esse associado aos seus maiores teores de argila, sem efeitos de ano e época.

As áreas não apresentaram diferenças significativas de densidade de partículas (Dp) na profundidade de 15-20 cm (Tabela 2), ficando os valores entre 2,61 e $2,69 \mathrm{~g} \mathrm{~cm}^{-3}$. Já na profundidade de 0 a $5 \mathrm{~cm}$ foram verificadas diferenças de Dp entre algumas áreas, com valores variando entre 2,40 e $2,57 \mathrm{~g} \mathrm{~cm}^{-3}$.

Observou-se aumento na densidade do solo (Ds) sob esplanadas em relação à mata de referência, principalmente na profundidade de 15-20 cm (Tabela 2). Os valores de Ds verificados nas esplanadas, na profundidade de $0-5 \mathrm{~cm}$, foram de 1,34 a 1,64 $\mathrm{g} \mathrm{cm}^{-3}$, aumentando, na profundidade de $15-20 \mathrm{~cm}$, para valores

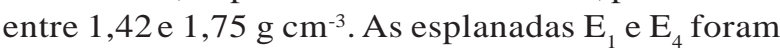
as que apresentaram os maiores valores de Ds em profundidade $\left(1,75 \mathrm{~g} \mathrm{~cm}^{-3}\right)$, o que pode estar relacionado ao fato de terem sido utilizadas na época chuvosa. Segundo Dias Júnior e Pierce (1996), o teor de água no momento em que o solo está sendo compactado tem grande influência na redução e redistribuição do espaço poroso. Os solos com baixos teores de água têm maior resistência à compactação. Kondo e Dias 
Tabela 1 - Caracterização física das áreas de mata e esplanadas, em amostras dos primeiros $20 \mathrm{~cm}$ do solo.

Table 1 - Physical characterization in the natural forest and cleared areas in the samples from the first $20 \mathrm{~cm}$ of soil.

\begin{tabular}{|c|c|c|c|c|c|c|c|c|}
\hline \multirow{2}{*}{\multicolumn{2}{|c|}{ Áreas }} & $\mathrm{AG}^{(1)}$ & $\mathrm{AF}^{(2)}$ & Silte & Argila & $\operatorname{ADA}^{(3)}$ & $\mathrm{IF}^{(4)}$ & $\mathrm{UCC}^{(5)}$ \\
\hline & & & & $\mathrm{g} \mathrm{kg}^{-1}$ & & & $\%$ & $\mathrm{~kg} \mathrm{~kg}^{-1}$ \\
\hline \multirow[b]{2}{*}{$A_{1}$} & $\mathrm{M}_{1}$ & 524 & 264 & 20 & 192 & 69,0 & $64 \mathrm{ab}$ & 0,16 с \\
\hline & $\mathrm{E}_{1}$ & 465 & 291 & 17 & 227 & 77,8 & $65 \mathrm{ab}$ & 0,17 bc \\
\hline \multirow{2}{*}{$A_{2}$} & $\mathrm{M}_{2}$ & 605 & 138 & 32 & 225 & 66,5 & $70 \mathrm{ab}$ & 0,17 bc \\
\hline & $\mathrm{E}_{2}$ & 512 & 183 & 35 & 270 & 94,3 & $65 \mathrm{ab}$ & $0,18 \mathrm{~b}$ \\
\hline \multirow{2}{*}{$\mathrm{A}_{3}$} & $\mathrm{M}_{3}$ & 467 & 294 & 37 & 202 & 77,2 & $61 \mathrm{ab}$ & 0,17 bc \\
\hline & $\mathrm{E}_{3}^{3}$ & 453 & 295 & 33 & 219 & 80,5 & $63 a b$ & $0,18 \mathrm{~b}$ \\
\hline \multirow{2}{*}{$\mathrm{A}_{4}$} & $\mathrm{M}_{4}^{3}$ & 483 & 299 & 18 & 200 & 81,6 & $59 \mathrm{~b}$ & 0,16 bc \\
\hline & $E_{4}^{4}$ & 472 & 293 & 17 & 218 & 82,9 & $57 \mathrm{~b}$ & 0,17 bc \\
\hline \multirow{2}{*}{$A_{5}$} & $\mathrm{M}_{5}$ & 483 & 174 & 36 & 307 & 71,8 & 76 a & 0,21 a \\
\hline & $\mathrm{E}_{5}$ & 459 & 182 & 39 & 320 & 79,6 & $75 \mathrm{a}$ & 0,22 a \\
\hline \multicolumn{2}{|l|}{$\mathbf{F}$} & $8,88^{*}$ & $14,61^{*}$ & $1,39^{\text {ns }}$ & $30,52^{*}$ & $1,61^{\mathrm{ns}}$ & $3,85^{*}$ & $41,11^{*}$ \\
\hline \multicolumn{2}{|c|}{ CV (\%) } & 8,04 & 18,50 & 24,89 & 9,26 & 21,47 & 13,42 & 4,98 \\
\hline
\end{tabular}

$\mathrm{M}=$ mata; $\mathrm{E}=$ esplanada; $\mathrm{F}=$ teste $\mathrm{F} ;$ e $\mathrm{CV}=$ coeficiente de variação. ${ }^{(1)}$ Areia grossa; ${ }^{(2)}$ areia fina; ${ }^{(3)}$ argila dispersa em água; ${ }^{(4)}$ índice de floculação; ${ }^{(5)}$ umidade na capacidade de campo; * significativo a 5\%; e ${ }^{\text {ns }}$ não significativo.

As médias seguidas das mesmas letras na coluna não diferem entre si, pelo teste de Tukey a 5\% de significância.

Tabela 2 - Densidade de partículas, densidade do solo, porosidade total e umidade do solo, nos primeiros $20 \mathrm{~cm}$, nas áreas de estudo.

Table 2 - Particle density, soil density, total porosity and soil moisture, in the first $20 \mathrm{~cm}$ in the areas of study.

\begin{tabular}{|c|c|c|c|c|c|c|c|c|c|}
\hline \multirow{2}{*}{\multicolumn{2}{|c|}{ ÁREAS }} & \multicolumn{2}{|c|}{ Densidade de partículas } & \multicolumn{2}{|c|}{ Densidade do solo } & \multicolumn{2}{|c|}{ Porosidade total } & \multicolumn{2}{|c|}{$\begin{array}{c}\text { Umidade do solo } \\
0-20 \mathrm{~cm}\end{array}$} \\
\hline & & $0-5 \mathrm{~cm}$ & $15-20 \mathrm{~cm}$ & $0-5 \mathrm{~cm}$ & $15-20 \mathrm{~cm}$ & $0-5 \mathrm{~cm}$ & $15-20 \mathrm{~cm}$ & Seca & Chuva \\
\hline & & \multicolumn{4}{|c|}{$\mathrm{g} \mathrm{cm}^{-3}$} & \multicolumn{2}{|c|}{$\mathrm{m}^{3} \mathrm{~m}^{-3}$} & \multicolumn{2}{|c|}{$\mathrm{kg} \mathrm{kg}^{-1}$} \\
\hline & $\mathrm{M}_{1}$ & 2,51 abc & 2,61 & 1,09 de & $1,12 \mathrm{~d}$ & $0,56 \mathrm{a}$ & $0,57 \mathrm{ab}$ & $0,06 \mathrm{~cd}$ & $0,10 \mathrm{f}$ \\
\hline & $\mathrm{E}_{1}$ & 2,57 a & 2,68 & 1,64 а $(50 \%)$ & 1,75 а $(56 \%)$ & 0,36 e & $0,35 \mathrm{e}$ & $0,06 \mathrm{~cd}$ & 0,11 ef \\
\hline & $\mathrm{M}_{2}$ & 2,47 abc & 2,65 & 1,11 de & $1,16 \mathrm{~d}$ & 0,55 a & $0,56 \mathrm{ab}$ & $0,08 \mathrm{bc}$ & $0,16 \mathrm{~cd}$ \\
\hline & $\mathrm{E}_{2}$ & 2,49 abc & 2,66 & 1,48 ab (33\%) & 1,59 b $(37 \%)$ & 0,40 de & $0,40 \mathrm{~d}$ & $0,06 \mathrm{~cd}$ & 0,15 cde \\
\hline \multirow[t]{2}{*}{$\mathrm{A}_{3}$} & $\mathrm{M}_{3}^{2}$ & $2,54 \mathrm{ab}$ & 2,63 & 1,18 cde & $1,19 \mathrm{~d}$ & $0,53 \mathrm{ab}$ & $0,55 \mathrm{ab}$ & $0,07 \mathrm{~cd}$ & 0,18 bc \\
\hline & $\mathrm{E}_{3}$ & 2,51 abc & 2,67 & 1,34 bc $(13 \%)$ & 1,42 с $(19 \%)$ & 0,46 bcd & $0,47 \mathrm{c}$ & $0,05 \mathrm{~d}$ & 0,17 bc \\
\hline \multirow{2}{*}{$\mathrm{A}_{4}$} & $\mathrm{M}_{4}$ & $2,52 a b$ & 2,63 & 1,26 bcd & $1,25 \mathrm{~d}$ & 0,50 abc & $0,52 \mathrm{~b}$ & $0,05 \mathrm{~d}$ & 0,13 def \\
\hline & $E_{4}^{4}$ & 2,48 abc & 2,68 & 1,43 b $(13 \%)$ & 1,75 а $(40 \%)$ & $0,42 \mathrm{~cd}$ & $0,34 \mathrm{e}$ & 0,08 bc & 0,15 cde \\
\hline \multirow{2}{*}{$\mathrm{A}_{5}$} & $\mathrm{M}_{5}$ & $2,40 \mathrm{c}$ & 2,69 & 1,00 e & $1,12 \mathrm{~d}$ & 0,58 а & 0,58 а & 0,11 a & 0,23 a \\
\hline & $E_{5}$ & 2,45 bc & 2,68 & 1,35 bc $(35 \%)$ & 1,45 с $(29 \%)$ & $0,45 \mathrm{~cd}$ & $0,46 \mathrm{c}$ & $0,10 \mathrm{ab}$ & $0,21 \mathrm{ab}$ \\
\hline \multicolumn{2}{|l|}{$\mathbf{F}$} & $4,61^{*}$ & $1,25^{\mathrm{ns}}$ & $18,82^{*}$ & $71,74^{*}$ & $17,63^{*}$ & $69,69^{*}$ & $12,78^{*}$ & $29,95^{*}$ \\
\hline \multicolumn{2}{|c|}{ CV (\%) } & 2,29 & 2,21 & 9,38 & 5,65 & 10,23 & 6,38 & 28,92 & 8,43 \\
\hline
\end{tabular}

$\mathrm{M}=$ mata; $\mathrm{E}$ = esplanada; $\mathrm{F}$ = teste $\mathrm{F} ;$ e $\mathrm{CV}$ = coeficiente de variação. Os percentuais entre parênteses representam o acréscimo na densidade do solo entre mata e esplanada. * significativo a 5\%; e ${ }^{\text {ns }}$ não significativo.

As médias seguidas das mesmas letras na coluna não diferem entre si, pelo teste de Tukey a 5\% de significância.

Júnior (1999), estudando a compressibilidade de três Latossolos na região de Lavras, MG, mostraram que o incremento do teor de água do solo provocou nesses solos redução na capacidade de suporte, independentemente do tipo de manejo a que haviam sido submetidos. Esses autores afirmaram que o teor de água do solo foi o principal fator regulador do comportamento compressivo desses solos. As esplanadas utilizadas no período da estiagem apresentaram as menores Ds nas profundidades avaliadas, sendo $E_{3}$ e $E_{5}$ aquelas em que se constataram densidades mais baixas.

A compacidade dos solos, por sua vez, altera a sua porosidade total (PT). Observou-se perda considerável da porosidade nos solos sob áreas de

Revista Árvore, Viçosa-MG, v.35, n.3, Edição Especial, p.737-744, 2011 
esplanadas, em comparação com os solos sob vegetação (Tabela 2). Os espaços porosos perdidos com o efeito da compactação são, na maioria, macroporos, que são importantes na movimentação de água e ar pelo solo. Com a diminuição da taxa de infiltração, há aumento no escoamento superficial, uma vez que a velocidade da água no perfil do solo depende do tamanho dos poros (LOPES et al., 2006).

As áreas de esplanadas apresentaram valores muito elevados de resistência à penetração (RP), e os testes evidenciaram incremento expressivo de resistência na época da seca (Figura 1), quando a umidade dos solos se encontrava bem abaixo da UCC deles (Tabelas 2 e 1),
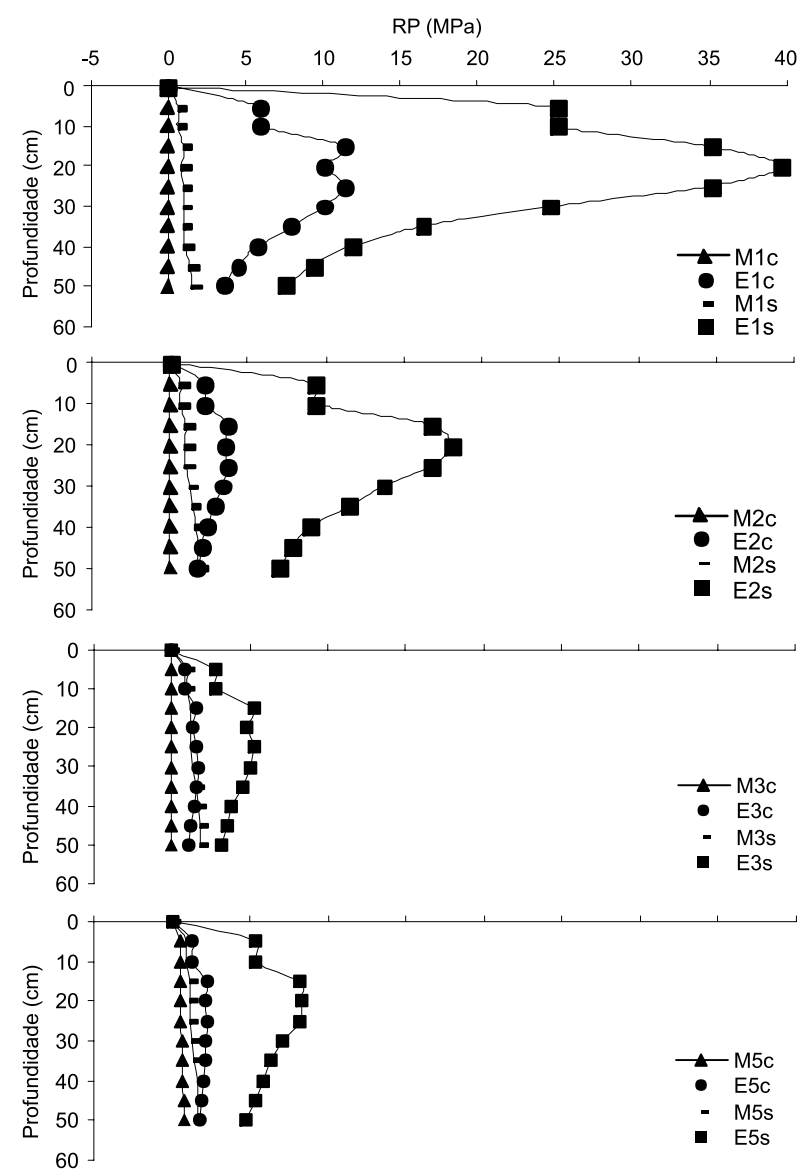

Figura 1 - Resistência do solo à penetração - testes realizados no período chuvoso (c) e período seco (s). $\mathrm{M}=$ mata; e E = esplanada.

Figure 1 - Soil penetration resistance - tests carried out in the rainy (c) and dry (s) season. $M$ = natural forest; and $E=$ cleared area . em relação à época chuvosa, quando a umidade do solo ficou próxima a UCC. Essa é uma tendência esperada e também constatada por Araújo et al. (2004) e Genro Júnior et al. (2004), que encontraram maiores valores de RP quando os solos encontravam-se mais secos e decréscimo linear da RP com o incremento da umidade.

Na época chuvosa, as áreas de mata praticamente não apresentaram resistência à penetração, e na época da seca os valores de RP sofreram ligeiro aumento. Os elevados valores de RP nas áreas de esplanadas podem ser associados à deposição de madeira e ao tráfego de máquinas, assim como constatado por Fernandes e Souza (2003), que verificaram diferenças expressivas de resistência à penetração antes e depois das passadas de máquinas, enquanto Lopes et al. (2006) registraram efeitos significativos na resistência à penetração em um solo de uso florestal. As esplanadas $E_{3}$ e $E_{5}$ apresentaram os menores valores de RP, concordando com os menores de Ds observados nesses espaços. Já $\mathrm{E}_{1}$ e $\mathrm{E}_{4}$ foram as que apresentaram os maiores valores de RP, tanto nos testes realizados na época da seca quanto na época chuvosa, resultados esses coerentes com os maiores valores de densidade do solo encontrados nessas áreas e provavelmente decorrentes do fato de a exploração nessas áreas ter sido efetuada na estação chuvosa.

O estado de agregação, expresso em diâmetro médio ponderado (DMP), indica considerável redução no tamanho médio dos agregados nas áreas sob esplanadas. Enquanto as áreas de mata apresentaram DMP médio de 1,92 $\mathrm{mm}$, as esplanadas exibiram valores médios de 1,01 mm, evidenciando que essa prática do manejo florestal afeta negativamente a estrutura do solo. As esplanadas $E_{3}$ e $E_{5}$ tiveram menor impacto sobre a estruturação do solo, decorrente da prática florestal em estudo, com DMP de 1,23 mm e 1,70 mm, respectivamente; já $\mathrm{E}_{1}$ e $\mathrm{E}_{4}$ apresentam as maiores deteriorações de estrutura do solo, com DMP de 0,52 mm e $0,75 \mathrm{~mm}$.

De modo geral, os solos das áreas estudadas demonstraram pobreza química (Tabela 3), com baixos percentuais de saturação por bases e altos percentuais de saturação por alumínio. Os valores de pH em água variaram de 3,6 a 4,2 nas áreas de mata e de 3,9 a 4,6 nas áreas de esplanadas, caracterizando acidez elevada. A acidez mais acentuada, verificada em algumas áreas de mata em relação às áreas de esplanada, pode ser 
atribuída ao efeito da matéria orgânica, que pode contribuir para incrementar a acidez do solo. Parte dessa acidez pode estar relacionada à liberação de ácidos orgânicos durante a decomposição da serapilheira (ALEXANDER; CRESSE, 1995).

Com a retirada da mata, verificou-se redução da CTC dos solos, o que pode ser associado à diminuição dos teores de matéria orgânica (Tabela 4). A retirada da vegetação também reduziu expressivamente os teores de potássio no solo, que predomina nos solos sob mata. Segundo Araújo et al. (1994), os maiores teores de matéria orgânica podem ser uma razão para maiores teores de $\mathrm{Ca}$ e $\mathrm{Mg}$.

Com relação ao fósforo, observaram-se maiores teores nas áreas sob mata, o que de acordo com Diez et al. (1991) se deve ao aporte contínuo de folhas que sofrem mineralização e contribuem para a manutenção do fósforo no solo. Os teores de P e Ca estão entre os nutrientes alterados pelos processos de derruba e queima (SILVA et al., 2007). Segundo Tome Júnior (1997), o P disponível tende a diminuir com a profundidade, acompanhando o teor de matéria orgânica.

Na comparação entre áreas de vegetação natural e de esplanada, observou-se redução nos teores de C-total e N-total com a retirada da mata (Tabela 4), exceto na área 4. Isso reflete o conhecido efeito da substituição de ecossistemas nativos por áreas manejadas, em que parte do $\mathrm{C}$ e $\mathrm{N}$ é perdida com a
Tabela 4 - Carbono orgânico total, nitrogênio total e carbono mineralizável nas áreas de estudo.

Table 4-Total organic carbon, total nitrogen and mineralizable carbon in the areas of study.

\begin{tabular}{|c|c|c|c|c|}
\hline \multirow{2}{*}{\multicolumn{2}{|c|}{ ÁREAS }} & $\mathrm{COT}^{(1)}$ & $\mathrm{NT}^{(2)}$ & \multirow{2}{*}{$\frac{\mathrm{C} \min ^{(3)}}{\mathrm{g} \mathrm{kg}^{-1}}$} \\
\hline & & \multicolumn{2}{|c|}{ dag $k^{-1}$} & \\
\hline \multirow{2}{*}{$\mathrm{A}_{1}$} & $\mathrm{M}_{1}$ & $1,07 \mathrm{~b}$ & 0,69 bc & 1,12 bc \\
\hline & $\mathrm{E}_{1}$ & $0,51 \mathrm{~cd}$ & $0,30 \mathrm{~d}$ & $0,36 \mathrm{c}$ \\
\hline \multirow{2}{*}{$\mathrm{A}_{2}$} & $\mathrm{M}_{2}$ & $0,97 \mathrm{~b}$ & 0,72 bc & $1,70 \mathrm{ab}$ \\
\hline & $\mathrm{E}_{2}$ & $0,51 \mathrm{~cd}$ & $0,35 \mathrm{~d}$ & $0,61 \mathrm{c}$ \\
\hline \multirow{2}{*}{$\mathrm{A}_{3}$} & $\mathrm{M}_{3}$ & $0,99 \mathrm{~b}$ & 0,69 bc & $1,76 \mathrm{ab}$ \\
\hline & $\mathrm{E}_{3}$ & 0,89 b & 0,62 с & $1,04 \mathrm{bc}$ \\
\hline \multirow{2}{*}{$\mathrm{A}_{4}$} & $\mathrm{M}_{4}$ & 0,77 bcd & $0,54 \mathrm{~cd}$ & $1,82 \mathrm{ab}$ \\
\hline & $\mathrm{E}_{4}$ & 0,87 bc & 0,66 с & $1,35 \mathrm{~b}$ \\
\hline \multirow{2}{*}{$\mathrm{A}_{5}$} & $M_{5}$ & 1,79 а & 1,21 a & 2,39 a \\
\hline & $\mathrm{E}_{5}$ & 1,50 a & $0,94 \mathrm{~b}$ & 2,09 a \\
\hline \multicolumn{2}{|l|}{$\mathbf{F}$} & $22,27^{*}$ & $25,11^{*}$ & $14,84^{*}$ \\
\hline \multicolumn{2}{|c|}{ CV (\%) } & 24,02 & 22,10 & 34,66 \\
\hline
\end{tabular}

$\mathrm{M}=$ mata $\mathrm{E}=$ esplanada; $\mathrm{F}=$ teste $\mathrm{F} ; \mathrm{e} \mathrm{CV}=$ coeficiente de variação. (1) carbono orgânico total; ${ }^{(2)}$ nitrogênio total; (3) carbono mineralizável por evolução de $\mathrm{CO}_{2}$ após 49 dias de incubação; * significativo a $5 \%$; e ns não significativo.

mineralização da matéria orgânica e quase sempre sem a devida reposição desta. Adicionalmente, perdas de carbono orgânico também podem ser atribuídas à destruição de macroagregados e subsequente mineralização do carbono orgânico lábil (ELLIOTT, 1986).

Os valores referentes à produção acumulada de $\mathrm{CO}_{2}$ (carbono mineralizável) em 49 dias de incubação (Tabela 4) demonstram maior evolução do gás nas áreas sob mata. A presença e acúmulo de restos vegetais

Tabela 3 - Características químicas do solo nas áreas estudadas.

Table 3 - Soil chemical characteristic in the areas of study.

\begin{tabular}{|c|c|c|c|c|c|c|c|c|c|c|c|c|}
\hline \multirow{2}{*}{ ÁREAS } & \multirow{2}{*}{$\mathrm{pH}$} & $\mathrm{P}$ & $\mathrm{K}$ & $\mathrm{Ca}^{2+}$ & $\mathrm{Mg}^{2+}$ & $\mathrm{Al}^{3+}$ & $\mathrm{H}+\mathrm{Al}$ & $\mathrm{SB}^{(1)}$ & $\mathbf{t}^{(2)}$ & $T^{(3)}$ & $\mathrm{V}^{(4)}$ & $\mathrm{m}^{(5)}$ \\
\hline & & \multicolumn{2}{|c|}{$\mathrm{mg} \mathrm{dm}^{-3}$} & \multicolumn{6}{|c|}{$\mathrm{cmol}_{\mathrm{c}} \mathrm{dm}^{-3}$} & & \multicolumn{2}{|c|}{$\%$} \\
\hline \multirow{2}{*}{$\mathrm{A}_{1}$} & $3,6 \mathrm{c}$ & $0,9 \mathrm{ab}$ & 16,0 abc & 0,0 & 0,1 & 1,2 & $5,8 \mathrm{ab}$ & 0,1 & $1,4 \mathrm{ab}$ & $5,9 \mathrm{ab}$ & $2,1 \mathrm{ab}$ & 91,0 \\
\hline & 4,6 a & $0,0 \mathrm{~b}$ & 0,0 с & 0,0 & 0,0 & 0,4 & $3,0 \mathrm{~b}$ & 0,0 & $0,5 \mathrm{~b}$ & $3,0 \mathrm{~b}$ & $1,0 \mathrm{~b}$ & 94,0 \\
\hline \multirow{2}{*}{$\begin{array}{ll}\mathrm{A}_{2} & \mathrm{M}_{2} \\
\mathrm{E}_{2}\end{array}$} & $3,7 \mathrm{c}$ & 1,0 a & $23,0 \mathrm{ab}$ & 0,0 & 0,1 & 0,7 & $4,4 \mathrm{ab}$ & 0,1 & $0,9 \mathrm{ab}$ & $4,6 \mathrm{ab}$ & $3,0 \mathrm{ab}$ & 84,3 \\
\hline & $4,5 \mathrm{ab}$ & $0,1 \mathrm{ab}$ & 0,0 с & 0,0 & 0,0 & 0,4 & $2,6 \mathrm{~b}$ & 0,0 & $0,4 \mathrm{~b}$ & $2,6 \mathrm{~b}$ & $1,2 \mathrm{~b}$ & 93,0 \\
\hline \multirow{2}{*}{$\mathrm{A}_{3}$} & 3,8 bc & $0,8 \mathrm{ab}$ & 17,0 abc & 0,0 & 0,1 & 0,7 & $4,4 \mathrm{ab}$ & 0,1 & $0,8 \mathrm{ab}$ & $4,6 \mathrm{ab}$ & $2,8 \mathrm{ab}$ & 84,5 \\
\hline & 3,9 bc & $0,5 \mathrm{ab}$ & 3,3 bc & 0,0 & 0,0 & 0,9 & $4,4 \mathrm{ab}$ & 0,1 & $1,0 \mathrm{ab}$ & $4,5 \mathrm{ab}$ & $1,4 \mathrm{~b}$ & 94,0 \\
\hline \multirow{2}{*}{$\begin{array}{l}\mathrm{M}_{4}^{3} \\
\mathrm{E}_{4}\end{array}$} & 4,2 abc & $0,7 \mathrm{ab}$ & 13,0 abc & 0,0 & 0,1 & 0,6 & $3,7 \mathrm{ab}$ & 0,1 & $0,7 \mathrm{ab}$ & 3,8 b & $3,2 \mathrm{ab}$ & 83,3 \\
\hline & 4,2 abc & $0,4 \mathrm{ab}$ & 4,0 bc & 0,0 & 0,0 & 0,9 & $3,8 \mathrm{~b}$ & 0,1 & $1,0 \mathrm{ab}$ & $3,9 \mathrm{~b}$ & $1,2 \mathrm{~b}$ & 95,4 \\
\hline \multirow{2}{*}{$A_{5}$} & 4,0 abc & $0,6 \mathrm{ab}$ & 30,0 a & 0,4 & 0,2 & 1,1 & 7,3 a & 0,7 & $1,8 \mathrm{a}$ & 8,0 a & 8,5 a & 62,6 \\
\hline & $4,4 \mathrm{ab}$ & $0,3 \mathrm{ab}$ & 15,3 abc & 0,2 & 0,1 & 0,5 & $5,0 \mathrm{ab}$ & 0,4 & $0,9 \mathrm{ab}$ & $5,4 \mathrm{ab}$ & $6,6 \mathrm{ab}$ & 62,0 \\
\hline $\mathbf{F}$ & $7,2^{*}$ & $3,5^{*}$ & $5,3^{*}$ & $2,8^{*}$ & $5,2^{*}$ & $3,1^{*}$ & $4,5^{*}$ & $3,5^{*}$ & $4,1^{*}$ & $4,6^{*}$ & $3,9^{*}$ & $3,1^{*}$ \\
\hline CV (\%) & 4,8 & 56,9 & 64,2 & 21,4 & 50,7 & 33,0 & 22,9 & 101,5 & 32,6 & 24,5 & 65,0 & 12,6 \\
\hline
\end{tabular}

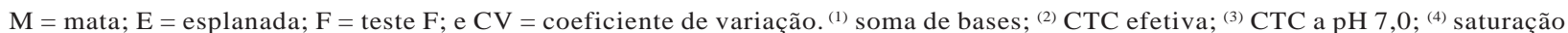
por bases; (5) saturação por alumínio; ${ }^{*}$ significativo a $5 \%$; e ${ }^{\text {ns }}$ não significativo.

As médias seguidas das mesmas letras na coluna não diferem entre si, pelo teste de Tukey a 5\% de significância.

Revista Árvore, Viçosa-MG, v.35, n.3, Edição Especial, p.737-744, 2011 
verificados na superfície do solo das áreas nativas proporcionaram melhorias ambientais na atividade microbiana.

\section{CONCLUSÃO}

1) As esplanadas representam um ambiente de solo extremamente degradado em suas propriedades físicas e químicas associadas ao carbono orgânico. A modificação na estrutura foi marcante em relação à mata, evidenciando-se um processo de compactação.

2) Não houve evidência de recuperação das propriedades físicas dos solos com o tempo de abandono das esplanadas, e a utilização de diferentes formas de esplanadas não proporcionou benefícios à qualidade do solo.

\section{AGRADECIMENTOS}

Ao CNPq, pelo apoio financeiro; e ao Governo do Estado de Mato Grosso, à Fazenda APASA e ao Sr. José Marcos Foloni, pelo apoio durante a pesquisa.

\section{REFERÊNCIAS}

ALEXANDER, C. E.; CRESSE, M. S. An assessment of the possible impacto $f$ expasion of native woodland cover on the chemistry of Scottish freshwaters. Forest Ecology and Management, v.73, n.1, p.1-27, 1995.

ARAÚJO, M. A. et al. Efeitos da escarificação na qualidade física de um Latossolo Vermelho distroférrico após treze anos de semeadura direta. Revista Brasileira de Ciência do Solo, v.28, n.4, p.495-504, 2004.

ARAÚJO, Q. K. et al. Ação da queima e da percolação sobre propriedades químicas de um Latossolo Vermelho-Amarelo variação una.

Revista Ceres, v.41, n.1, p.537-558, 1994.

DEFELIPO, B. V.; RIBEIRO, A. C. Análise química do solo: metodologia. Viçosa, MG: Universide Federal de Viçosa, 1981. 15p.

DIAS JÚNIOR, M. S.; PIERCE, F. J. O processo de compactação do solo e sua modelagem. Revisão de Literatura. Revista Brasileira de Ciência do Solo, v.20, n.1, p.175-182, 1996.
DIEZ, J. A. et al. Influência do pousio e da pastagem sobre a dinâmica de nutrientes em Oxissolos recentemente desflorestados na Amazônia Oriental. Pesquisa Agropecuária Brasileira, v.26, n.1, p.77-83, 1991.

ELLIOTT, E. T. Aggregate structure and carbon, nitrogen, and phosphorus in native and cultivated soils. Soil Science Society of America Journal, v.50, n.4, p.627-633, 1986.

EMPRESA BRASILEIRA DE PESQUISA AGROPECUÁRIA - EMBRAPA. Centro Nacional de Pesquisa de Solos. Manual de métodos de análises de solo. Rio de Janeiro: EMBRAPA/CNPS, 1997. v.1. 210p.

FERNANDES, H. C.; SOUZA, A. P. Compactação de um Latossolo Vermelho causada pelo tráfego do "forwarder". Revista Árvore, v.27, n.3, p.279-284, 2003.

FUNDAÇÃO ARTHUR BERNARDES - FUNARBE - Sistema para análises estatísticas SAEG, versão 9.0. Viçosa, MG: 2004.

GENRO JÚNIOR, S. A.; REINERT, D. J.; REICHERT, J. M. Variabilidade temporal da resistência à penetração de um Latossolo argiloso sob semeadura direta com rotação de culturas.

Revista Brasileira de Ciência do Solo, v.28, n.3, p.477-484, 2004.

GREGORICH, E. G.; VORONEY, R. P.;

KACHANOSK, I. Turnover of carbon through the microbial biomass in soils with different textures toxicity in subsoils. Soil Science Society of America Journal, v.50, n.1, p.28-34, 1990.

KONDO, M. K.; DIAS JÚNIOR, M. S.;

Compressibilidade de três Latossolos em função da umidade e uso. Revista Brasileira de Ciência do Solo, v.23, n.2, p.211-218, 1999.

KÖPPEN, W. P. Climatologia: con un estudio de los climas de la tierra. Mexico: Fondo de Cultura Economica, 1948. 478p.

LIMA, C. C. Dinâmica de carbono, características químicas do solo, sistema radicular e produção do feijoeiro em resposta à adição de composto à base de dejetos suínos. 1996. 114f. Dissertação (Mestrado em Solos e Nutrição de Plantas) Universidade Federal de Viçosa, Viçosa, MG, 1996. 
LOPES, S. E. et al. Compactação de um solo de uso florestal submetido ao tráfego de arraste de madeira. Revista Árvore, v.30, n.3, p.369-376, 2006.

RUIZ, H. A. Notas de aula. Disponível em: http://solos.ufv.br/sol640. Acessado em 20 de agosto de 2003.

RUIZ, H. A.; FERREIRA, G. B.; PEREIRA, J. B. Estimativa da capacidade de campo de Latossolos e Neossolos Quartzarênicos pela determinação do equivalente de umidade. Revista Brasileira de Ciência do Solo, v.27, n.2, p.389-393, 2003.

SILVA, R. C. et al. Alterações nas propriedades químicas e físicas de um chernossolo com diferentes coberturas vegetais. Revista Brasileira de Ciência do Solo, v.31, n.1, p.101-107, 2007.
STOLF, R. Impact penetrometer stolf model: data manipulation program version 2.1 . us Araras, Universidade Federal de São Carlos, 1991. (Software).

TOME JÚNIOR, J. B. Manual para interpretação de análise de solo. Guaíba: Agropecuária, 1997. 247p.

VALVERDE, S. R. et al. Evolução da participação do setor florestal na economia brasileira. In: CONGRESSO FLORESTAL BRASILEIRO, 8., 2003, São Paulo. Anais... São Paulo: SBS/SBEF, 2003. v. 2. CD ROM.

YEOMANS, J. C., BREMNER, J. M. A rapid and precise method for routine determination of organic carbon in soil. Communications Soil Science and Plant Analysis, v.19, p.1467-1476, 1988. 